\title{
PLASTIA Y ALARGAMIENTO DE TENDÓN ROTULIANO MEDIANTE ALAMBRE PUENTE EN RÓTULA Y COSECHA AUTÓLOGA DE SEMITENDINOSO Y GRACILIS. REPORTE DE CASO CLÍNICO. HOSPITAL POLICÍA NO.2 DE GUAYAQUIL, FEBRERO 2018
}

PLASTY AND LENGTHENING OF THE PATELLAR TENDON USING PATELLA BRIDGE WIRE AND AUTOLOGOUS HARVEST OF SEMITENDINOSUS AND GRACILIS. A CLINICAL CASE REPORT. POLICE HOSPITAL NO. 2 OF GUAYAQUIL, FEBRUARY 2018

PLASTIA E ALONGAMENTO DO TENDÃO PATELAR COM FIO PONTE PATELAR E RETIRADA AUTÓLOGA DO SEMITENDÍNEO E DO GRACILIS. RELATO DE CASO CLIINICO. HOSPITAL DA POLÍCIA NN 2 DE GUAYAQUIL, FEVEREIRO DE 2018

\section{CARLA GRAU MUÑOZ' ${ }^{1}$ XAVIER SÁNCHEZ ALARCÓN², PABLO VELIZ FUENTES², GONZALO QUILLUPANGUI NAVARRO³}

\author{
${ }^{1}$ Universidad Católica de Santiago de Guayaquil, Guayaquil, Ecuador \\ 2 Hospital de la Policía Nacional de Guayaquil №2, Guayaquil, Ecuador \\ ${ }^{3}$ Universidad Christian Albrechts, kiel, Alemania
}

\begin{abstract}
RESUMEN
Las lesiones del tendón rotuliano son casos poco frecuentes que suceden en su mayoría por lesiones traumáticas graves muchas veces apoyadas por factores concomitantes. Debido a ello, los resultados funcionales dependen de la calidad y la técnica de intervención quirúrgica. Sin embargo, no se puede anticipar la evolución de los elementos anatómicos comprometidos, pero sí dar un control posoperatorio correcto. Se presenta un paciente masculino de 35 años sin antecedentes mórbidos, evaluado ambulatoriamente con rodilla derecha en extensión $180^{\circ}$ y flexión $0^{\circ}$ como secuela postraumática e iatrogénica, con atrofia del cuádriceps femoral y dificultad para la marcha. Estudios imagenológicos evidenciaron hemiprótesis patello femoral, alambre rótula tibia, puente exostósico tibio rotuliano, tres alambres circulares en tubérculo anterior de la tibia. Se diagnostica anquilosis postraumática de rodilla derecha. Se planifica técnica de Kelikian modificada (injerto autólogo de rodilla opuesta más cosecha de Gracillis y cerclaje de alambre transpatellar). A las 3 semanas del postoperatorio, se evidencia $45^{\circ}$ de flexión y $180^{\circ}$ de extensión en rodilla derecha. Se concluye que la técnica de Kelikian modificada es una alternativa quirúrgica óptima en pacientes con anquilosis postraumática de rodilla.

PALABRAS CLAVE: tendón rotuliano, reconstrucción, alargamiento, cerclaje de alambre transpatellar, injerto autólogo.
\end{abstract}

ABSTRACT

Patellar tendon injuries are rare cases that mostly occur due to severe traumatic injuries, many times supported by concomitant factors. Because of this, functional results depend on the quality and technique of surgical intervention. However, the evolution of the compromised anatomical elements cannot be advanced, but correct postoperative control can be given. A 35-year-old male patient with no morbid history is presented, evaluated out patiently with a right knee in $180^{\circ}$ extension and $0^{\circ}$ flexion as a post-traumatic and iatrogenic sequel, with atrophy of the quadriceps femoris and difficulty walking. Imaging studies showed patellofemoral hemiprosthesis, tibia patella wire, patellar tibial exostosis bridge, three circular wires in the anterior tubercle of the tibia. Post-traumatic ankylosis of the right knee was diagnosed. Modified Kelikian technique (autologous opposite knee graft plus Gracillis harvest and transpatellar wire cerclage) was planned. At 3 weeks postoperatively, was evidenced a $45^{\circ}$ of flexion and $180^{\circ}$ of extension in the right knee. It concluded that the modified Kelikian technique is an optimal surgical alternative in patients with post-traumatic knee ankylosis. Keywords: Patellar tendon. Reconstruction. Elongation. Transpatellar wire cerclage. Autologous graft.

KEYWORDS: patellar ligament, reconstruction, lengthening, transpatellar wire cerclage, autologous graft.

Lesões do tendão patelar são casos raros que ocorrem principalmente por lesões traumáticas graves, muitas vezes apoiadas por fatores concomitantes. Por isso, os resultados funcionais dependem da qualidade e da técnica da intervenção cirúrgica. Entretanto, a evolução dos elementos anatômicos comprometidos não pode ser antecipada, mas o controle pós-operatório correto pode ser dado. É apresentado um paciente do sexo masculino, 35 anos, sem história mórbida, avaliado ambulatorialmente com joelho direito em extensão de $180^{\circ}$ e flexão de $0^{\circ}$ como sequela pós-traumática e iatrogênica, com atrofia do quadríceps femoral e dificuldade de deambulação. Os estudos de imagem mostraram hemiprótese patelo femoral, fio de tíbia patela, ponte de exostose tibial patelar, três fios circulares no tubérculo anterior da tíbia. Foi diagnosticada anquilose pós-traumática do joelho direito. A técnica Kelikiana modificada (enxerto autólogo do joelho oposto mais colheita de Gracillis e cerclagem transpatelar com fio) está planejada. Na $3^{a}$ semana de pós-operatório, foram evidenciados $45^{\circ}$ de flexão e $180^{\circ}$ de extensão no joelho direito. Conclui-se que a técnica Kelikiana modificada é uma alternativa cirúrgica ideal em pacientes com anquilose pós-traumática do joelho.

PALAVRAS-CHAVE: ligamento patelar, reconstrucao, alongamento, cerclagem de fio transpatelar, enxerto autólogo. 
INTRODUCCIÓN

Las lesiones en el tendón rotuliano son poco frecuentes, ${ }^{1,2}$ su incidencia es heterogénea ${ }^{3,4} y$ suceden en su mayoría por causas extrínsecas que tienen como característica la contracción forzada del músculo cuádriceps cuando la rodilla está flexionada. ${ }^{5,6}$. Aunque están asociadas también a enfermedades con concomitantes o iatrogénica ${ }^{7,8}$ que predisponen a una mala calidad de las fibras tendinosas que podrían predisponer una ruptura del aparato extensor en condiciones fisiológicas. ${ }^{2}$

En el caso de ser lesiones agudas, es mejor su tratamiento quirúrgico lo antes posible para evitar la retracción del tendón con ascenso consecuente de patela. ${ }^{8}$ En este tipo de lesión es característica la incapacidad de extensión de la rodilla, y para su corrección se aplican procedimientos como: suturas transóseos, cerclaje de alambre, cable de acero, suturas de alta resistencia absorbibles y no absorbibles, con ayuda de aloinjertos, autoinjertos, anclajes óseos, etc; ;,9 que en cierta medida actúan eficazmente en la recuperación de los arcos de movimiento. Sin embargo, no se puede escapar de las limitaciones de los procedimientos ni de evolución de los elementos anatómicos comprometidos en la articulación. ${ }^{11}$ Uno de ellos es la fibrosis del tendón rotuliano, así como estructuras adyacentes que en este caso impediría su funcionamiento normal, ya sea con incapacidad de flexión si queda con rótula baja o de extensión si es alta. ${ }^{7}$ El problema surge cuando no hay una técnica quirúrgica que sirva como Gold Estándar en el tratamiento de esta patología.

Es por ello que el presente reporte de caso se centra en la reconstrucción y alargamiento del tendón rotuliano mediante la técnica de sutura de alambre transpatelar e injerto autólogo de semitendinoso y gracilis en paciente con antecedentes de ruptura del tendón patelar que presenta incapacidad en la flexión con el objetivo de que sirva de guía para casos similares.

CASO CLÍNICO

Paciente masculino de 35 años, cuya ocupación es de policía. Presenta antecedentes desde hace ocho años tras sufrir un accidente de moto particular en la que cayó de un puente a una altura de ocho metros, resultando politraumatizado grave. Tuvo trauma craneoencefálico y fractura expuesta grave de rótula( multifragmentaria), tibia derecha así como de humero proximal derecho. Estuvo un mes inconsciente en UCI. Se le realizaron varias intervenciones quirúrgicas que incluyeron reduc- ción y osteosíntesis de fémur, humero proximal y tibia derecha, además de un cerclaje de rotula. Después de un año, debido a rodilla en extensión y deformidad, se ejecutó una artrolisis en la cual se rompió el cerclaje previo de la rótula; por ello se realizó uno nuevo.

Dos años después del accidente, el paciente presentaba rotula baja con limitación a la flexión (solo flexionaba $30^{\circ}$ ) acompañada de dolor, gonartrosis postraumática de rodilla derecha por lo que el traumatólogo a cargo interviene quirúrgicamente con artroscopia para eliminar adherencias. Al año siguiente de la última intervención, el paciente acude al traumatólogo porque su condición física no era estable; limitación total a la flexión y dificultad para la marcha. Con diagnóstico de artrosis patelo femoral derecha, se le realiza una nueva intervención para colocación de hemi prótesis patelo femoral derecha, con hallazgos de anquilosis patelo femoral, fibrosis, patela baja y con la complicación de tendón rotuliano corto. Después de todo lo descrito, el paciente continuó con su rodilla en extensión e incapaz de flexionar.

En febrero del 2018, acudió al servicio de Traumatología presentando tumefacción en rodilla derecha, atrofia del cuádriceps femoral, incapacidad para subir escaleras y dificultad a la marcha. Al examen físico se observó retracción del tendón rotuliano y desplazamiento consecuente de la rótula hacia superior, rodilla derecha en extensión $180^{\circ}$ y flexión $0^{\circ}$. No se pudo medir el Índice Insall Salvatti en la rodilla lesionada por estar en extensión, pero la rodilla no lesionada presentó un índice de 1.2. En la resonancia magnética se observan tres alambres ubicados en el tubérculo anterior de la tibia y hemi prótesis cóndilo femoral rotuliana (Figura 1 y 2).

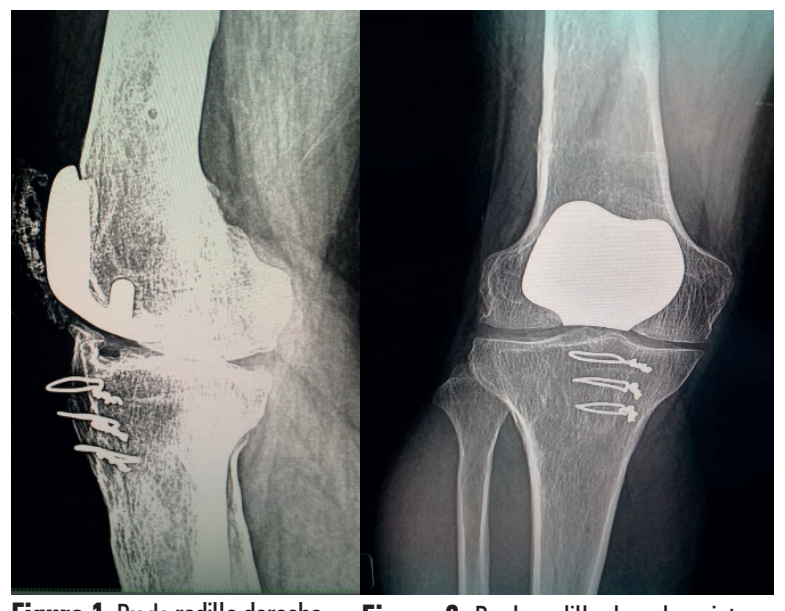

Figura 1. Rx de rodilla derecha, vista lateral Figura 2. Rx de rodilla derecha, vista
frontal 
Se programó para operarlo ya que la historia de fracaso en los tratamientos aplicados para solucionar el problema con su rodilla en extensión no tuvo resultados satisfactorios, quizás porque los profesionales que lo trataron aplicaron tratamientos que eran fáciles y seguros para los médicos, pero no efectivos para el paciente. Prefirieron quedarse en la zona de confort y comodidad, en lugar de adentrarse en la zona de reto y desarrollo dando un paso adelante para solucionar los problemas. Cuando más nos adentramos en la zona de reto y desarrollo es cuando se produce el aprendizaje y el desarrollo profesional como personal.

Después de realizar estudios anatómicos: reconstruir aparato extensor formado por cóndilo femoral, rótula, tendón rotuliano; biomecánicos: aparato extensor que venciendo gravedad realiza extensión de rodilla;11 y biológicos: tiempo de reparación y cicatrización con reemplazo del semitendinoso y Gracilis. Se decide realizar una reconstrucción y alargamiento del tendón rotuliano con cosecha de aloinjerto autólogo del Semitendinoso y Gracilis del lado contrario (Figura 3).
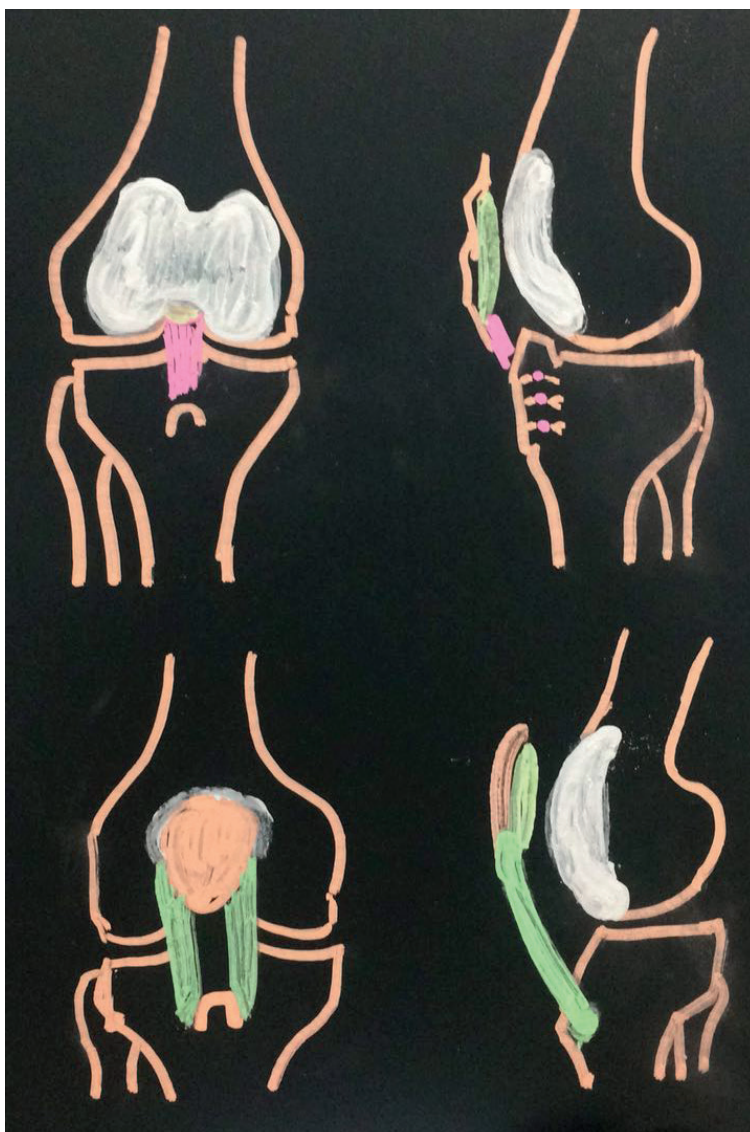

Figura 3. Planificación reconstrucción y alargamiento del tendón rotuliano con cosecha de aloinjerto autólogo del Semitendinoso y Gracilis del lado contrario.
TÉCNICA QUIRÚRGICA

Bajo anestesia con bloqueo peridural y raquídeo (combinado). Se coloca al paciente en decúbito supino en mesa quirúrgica radio lúcida, asepsia de miembros inferiores. Se colocan campos clínicos estériles. Primero con ayuda de un tenótomo se realiza cosecha de $17 \mathrm{~cm}$ del tendón obtenido del Semitendinoso y $24 \mathrm{~cm}$ del tendón obtenido del Gracilis de la pata de ganso del lado contrario. Luego se sutura por planos y con vicril 2.0 se sutura de manera longitudinal uniendo ambos tendones.

En la pierna derecha, y con ayuda de un marcador estéril, primero se ubica el área quirúrgica intentando seguir la cicatriz previa. Luego se procede a realizar una incisión longitudinal pararotuliana medial desde el tercio proximal del muslo hacia el tercio proximal de la pierna, para lograr exponer el tendón del cuádriceps, rótula hasta tubérculo anterior de la tibia. Se cauterizan vasos sangrantes. En este caso no se utilizó torniquete neumático y se profundizó por planos.

Se localiza el aparato extensor. Se encuentra con fibrosis y acortamiento del tendón rotuliano, fibrosis capsular anterior y stop óseo en tubérculo anterior de la tibia. Se procede a limpiar la fibrosis y se retiran tres alambres ubicados en tubérculo anterior de la tibia. Se efectúa una capsulotomía lateral, medial, anterior. Se secciona el alerón patelar externo. Se resecan los bordes de la rótula y coloca cera para hueso. Luego se tuneliza el tercio superior de la rótula y el tubérculo anterior de la tibia, para a continuación colocar un alambre puente de $1,8 \mathrm{~mm}$ que una ambas estructuras (Figura 4).

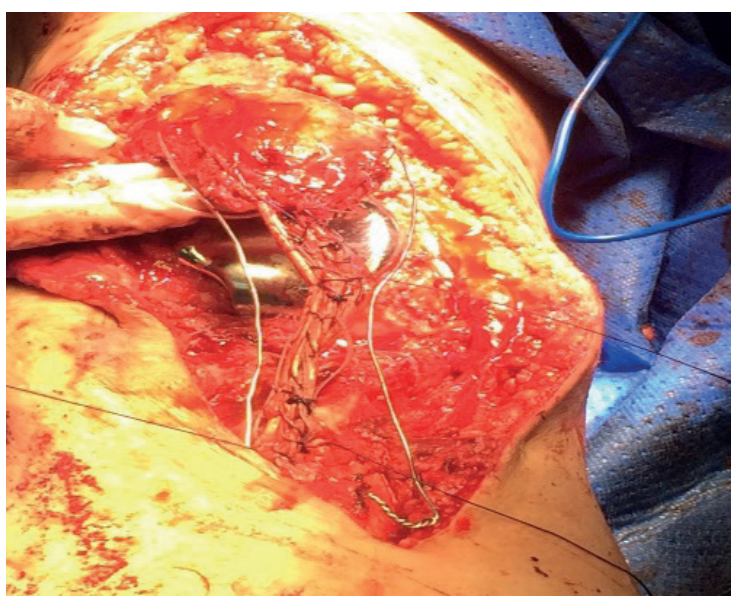

Figura 4. Se observa cómo se tunelizó el tercio superior de la rótula y el tubérculo anterior de la tibia para a continuación colocar un alambre puente de $1,8 \mathrm{~mm}$ que una ambas estructuras. También se tunelizó el tercio antero inferior de la rótula y tubérculo anterior de la tibia por debajo del túnel previo para pasar el tendón obtenido de la cosecha. 
Para garantizar la altura correcta de la patela mediante el cerclaje, se utilizó el criterio de Labelle y Laurin, que consiste en "una tangente aplicada en la parte anterior del fémur toca la base de la rótula con una flexión de la rodilla de 90 grados". ${ }^{11}$ Los túneles se fijaron con tornillos transferenciales, el proximal y distal de titanio con $7 \mathrm{~mm}$ diámetro y 2,5 de longitud. Después, se tuneliza el tercio antero inferior de la rótula y tubérculo anterior de la tibia por debajo del túnel previo para pasar el tendón obtenido de la cosecha. Este se pasa gracias a una aguja guía de medial a lateral en TAT y en dirección contraria en el caso de la patela. Los túneles aquí se fijan: en la rótula el tornillo transferencial de titanio, en la tibia tornillo transferencial de hidroxiapatita, de la misma longitud que los ya citados.

Se limpia el área quirúrgica con abundante solución salina y se sutura por planos dejando un dren de HEMOVAC de un cuarto de diámetro fijado a la pierna (Figura 5). En seguida se colocan apósitos y vendajes estériles. Primero Huata y luego vendas elásticas. Ulteriormente Brace a $30^{\circ}$ de extensión y $30^{\circ}$ de flexión.
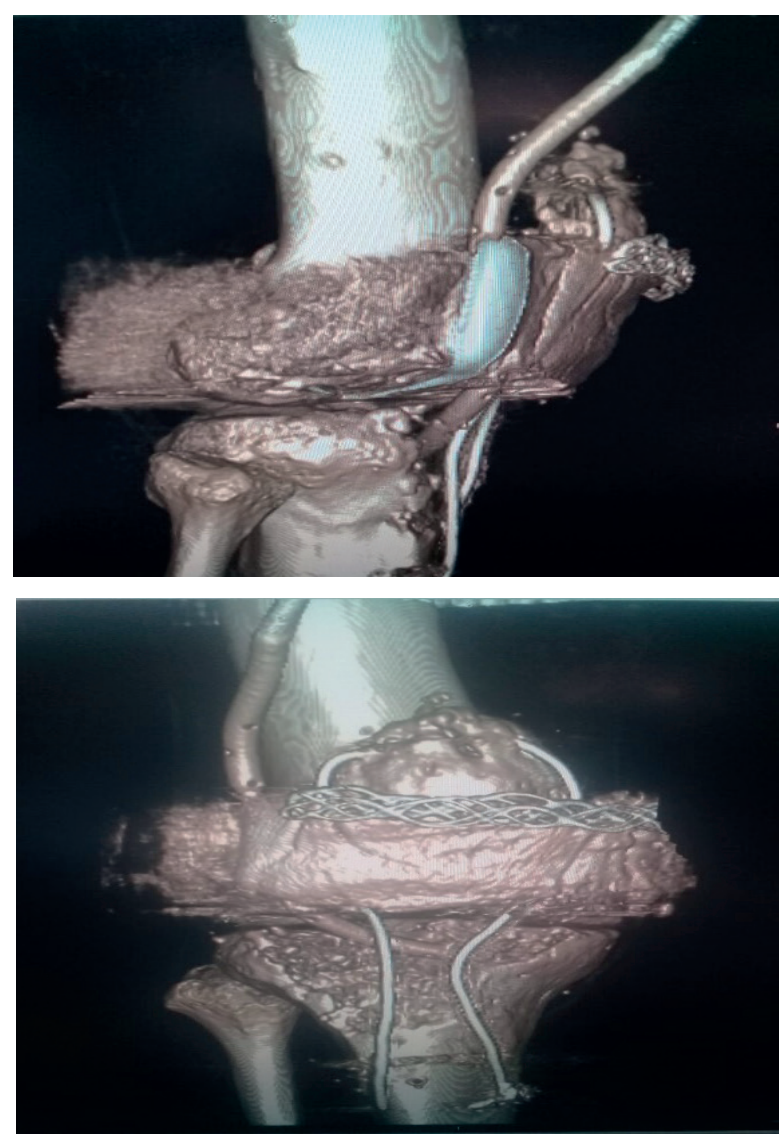

Figura 5. Se observa resonancia magnética de rodilla derecha vista ap y lateral, con alambre puente en la rótula reforzando con clavos; además se observa HEMOVAC

\section{POSOPERATORIO}

En dos semanas se retiran los apósitos y vendajes para limpiar la superficie y retirar puntos del área expuesta y colocar gasa estéril. Además, se observa queloide en miembro inferior derecho. El paciente permanece con el Brace a $30^{\circ}$ de flexión durante 2 semanas, al término de las cuales se le da el alta médica y se recomienda seguir con el inmovilizador durante 3 semanas más, y realizar ejercicios de flexión progresiva que no supere los $90^{\circ}$ grados para evitar la rotura de los injertos. A la tercera semana logra $45^{\circ}$ de flexión aproximadamente con $180^{\circ}$ de extensión (Figura 6).

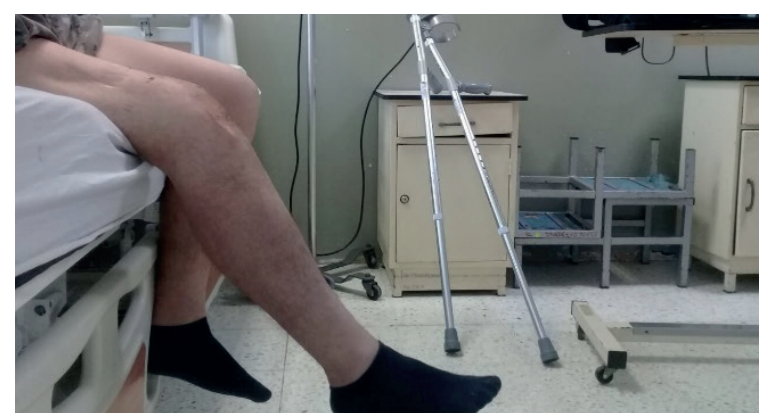

Figura 6. Paciente permanece con el Brace a $30^{\circ}$ de flexión durante 2 semanas.

\section{DISCUSIÓN}

El procedimiento en pacientes con antecedentes de lesión de ligamento rotuliano e intervenidos repetidas veces quirúrgicamente en el área de la articulación de la rodilla, conlleva, como resalta Rodríguez et al ${ }^{3}$ primero a un "desbridamiento debido a que la calidad ligamentosa y de tejidos blandos se va a ver claramente deteriorada" porque la magnitud del daño va a ser directamente proporcional al grado de limitación articular, en este caso su flexión. ${ }^{12}$ Por ello, en la técnica quirúrgica se procedió primero a realizar una capsulotomía medial, anterior y lateral, así como la limpieza de la fibrosis encontrada en la rótula y el propio ligamento patelar. Todo esto, más la liberación del alerón lateral a través de su sección, permitió eliminar la rigidez de la articulación. ${ }^{13}$

Se decidió llevar a cabo los túneles óseos con anclas debido a que es un método más efectivo para lograr una integración osteotendinosa fuerte, con un postoperatorio más corto y sin lugar a remoción de material. ${ }^{14,15}$ Además prepara el lecho de inserción del semitendinoso y el cerclaje de alambre que junto al anclaje, reforzarán a la articulación. ${ }^{12,14}$

La técnica de Kelikian, que se basa en la utilización del autoinjerto de semitendinoso para crear un marco sobre sí mismo en su inserción distal, al 
atravesar los dos túneles transóseos el de la tibia y la rótula se ve modificado al utilizar un injerto autólogo de la rodilla opuesta más cosecha de Gracilis. ${ }^{3}$ Fue necesaria la utilización de este tipo de plastia ya que el tejido ligamentoso rotuliano estará debilitado. ${ }^{3}$ En este caso, se descartó el uso de aloinjertos, fue suficiente con la longitud obtenida cosecha realizada y no se han presentado complicaciones observables ni disminución de la fuerza muscular como en un momento afirmaba Matus. ${ }^{16}$ Es importante recordar que en el procedimiento quirúrgico se debe realizar una correcta hemostasia en todo momento para evitar incomodidades con el dolor posoperatorio.

En la presente intervención se retiró la totalidad del tendón, pero para próximos procedimientos sería recomendable preservar su inserción distal ya que como afirma Chen, et al ${ }^{17}$ al conservar su aporte sanguíneo evita la necrosis que se podría producir con un injerto libre. Cabe recalcar que no solo se puede realizar injertos diferentes al semitendinoso. ${ }^{1,15}$

El cerclaje de alambre tiene como objetivo proteger la reconstrucción del ligamento realizada así como fijar y direccionar de manera adecuada la rótula, para evitar una patela alta o baja., ${ }^{3,14}$ Hay que tener en cuenta y evitar un exceso de tensión en el cerclaje porque puede producir como consecuencia principal una rótula baja. ${ }^{3},{ }^{7}$ Además, para una correcta altura de la rótula hay que tener en cuenta ya sea el criterio de Labelle y Laurin o el índice Insall Salvatti con ayuda de radiografías in situ. ${ }^{11,18}$ La desventaja de esta técnica de aumentación es que luego se tendrá que realizar una segunda intervención para el retiro de alambre.,14

Después del procedimiento quirúrgico, es necesario la monitorización del paciente con ejercicios isométricos de rehabilitación a partir de las dos semanas para evitar la contractura muscular tomando en cuenta la biomecánica de la rodilla. ${ }^{13}$ También, es correcto realizar ejercicios de flexión progresiva suaves para que no deterioren los ligamentos fijados. Pues como afirma Álvarez, et al. ${ }^{12}$ el comienzo del proceso de rehabilitación lo antes posible después de este tipo de procedimiento quirúrgico, ayuda a la prevención de una nueva artrofibrosis en la articulación de la rodilla.

\section{CONCLUSIÓN}

Se concluye que la reconstrucción y alargamiento del tendón rotuliano mediante la técnica de sutura de alambre transpatelar e injerto autólogo de
Semitendinoso y Gracilis del lado contralateral (Técnica de Kelilian modificada), es una buena y reproducible técnica quirúrgica en pacientes con antecedentes de ruptura crónica del ligamento patelar que, debido a la fibrosis y retracción del mismo, provocan anquilosis de rodilla. se evidenció recuperación estructural y de los arcos de movilidad a las pocas semanas del posoperatorio.

\section{REFERENCIAS BIBLIOGRÁFICAS}

1. Rupturas del aparato extensor de la rodilla y fracturas de la rótula - ScienceDirect [Internet]. Disponible en: https://www.sciencedirect.com/ science/article/pii/S2211033X18878926

2. Ruptura traumática aguda del tendón del cuádriceps tratado con sutura de alambre transpatelar Gac Méd Espirit vol.19 no.1 Sancti Spíritus ene.-abr. 2017. [Internet]. Disponible en: http:// scielo.sld.cu/scielo.php?script=sci_arttext\&pid $=$ S1608-89212017000100012

3. Álvarez JR, Castellano J, Macías SM, Moreno CGM, Cabrera AC, Navarro RN. Roturas del aparato extensor de la rodilla. En 2006.

4. Marqueta PM, Tarrero LT. * Médico de la Selección Española Absoluta "B" Femenina de Baloncesto. Secretario General de la Federación Española de Medicina del Deporte. ${ }^{* *}$ Médico de la Selección Española Junior Femenina de Baloncesto. :5.

5. Ciccarello VA, Kaltman B. Reconstrucción y restauración de la rotura aguda del tendón rotuliano con aumento del tendón semitendinoso preservando su inserción distal: Reporte de un caso. Rev Asoc Argent Ortop Traumatol. noviembre 2016;81(4):315-9.

6. Las lesiones por sobrecarga en las extremidades inferiores desde el punto de vista biomecánico | Fernando Pifarré San Agustin | Request PDF [Internet]. Disponible en: https://www.researchgate.net/publication/301277725_Las_lesiones_por_sobrecarga_en_las_extremidades_inferiores_desde_el_punto_de_vista_biomecanico

7. Ca E-M, Ga G-E. Tratamiento de la rotura no reciente del tendón patelar con tendones semitendinoso y gracilis. Acta ortopédica Mex. 6 .

8. Marín J, Posada A. Técnica para la reconstrucción de rupturas crónicas del tendón rotuliano, Rev. Col de Or Tra, Vol. 21 Núm.3. Septiembre 2007 Pags. 200-202. [Internet]. Disponible en: http://www.imbiomed.com/1/1/ articulos.php? method=showDetail\&id articulo $=48590 \&$ id_seccion $=2791 \&$ id $_{-}$ ejemplar $=4923 \& i d \_r e v i s t a=82$

9. Druskin SC, Rodeo SA. Novel Treatment of a Failed Quadriceps Tendon Repair in a Diabetic 
Patient Using a Patela-Quadriceps Tendon Allograft. HSS J. 2013 Jul; 9(2): 195-199.

10. Pruna R, Artells R. Cambios conceptuales en la medicina deportiva actual. :2.

11. Bauer R, Kerschbaumer F, Poisel S. Cirugía Ortopédica Rodilla. Primera Edición. Vol. 2. Madrid: Márbán; 2004. 374 p.

12. López AÁ, Lorenzo YG, Álvarez AP, Lorenzo MG. Artrofibrosis de la rodilla. Arch Méd Camagüey [Internet]. 4 de septiembre de 2014;14(4). Disponible en: http://www.revistaamc.sld.cu/index. php/amc/article/view/2335

13. Sems SA, Vaquero J, Sociedad Española de Cirugía Ortopédica y Traumatología, American Academy of Orthopaedic Surgeons. Fracturas de la rodilla. Madrid: Editorial Médica Panamericana; 2010.

14. Allende PD, López AC, Acevedo AP, León JHD, Echazabal JH. Técnica de reconstrucción en roturas recidivantes del tendón patelar. Rev Chil Ortop Traumatol. agosto de 2017;58(2):59-65.
15. Ruptura del tendón rotuliano en deportistas - Tratamiento con anclajes óseos - Revista de Artroscopía [Internet]. Disponible en: https://www.revistaartroscopia.com/ ediciones-anteriores/2009/volumen-16-numero-2/24-volumen-05-numero-1/ volumen-16-numero-2/584-ruptura-del-tendonrotuliano-en-deportistas-tratamiento-conanclajes-oseos

16. Matus J, Martínez H. Tratamiento de lesión de tendón rotuliano no reciente. Reporte de caso. Acta Ortopédica Mex. 2010; 24(6): Nov.-Dic: 412-419.

17. Chen B, Li R, Zhang S. Reconstruction and restoration of neglected ruptured patelar tendon using semitendinosus and gracilis tendons with preserved distal insertions: two case reports. The Knee. agosto de 2012;19(4):508-12.

18. Fuentes $S$. Ruptura crónica de tendón rotuliano en adolescente: Presentación de un caso. Vol. 15, Núm. 2, julio-diciembre 2013, pp. 118-122. 